ÉGYPTE

monde arabe

\section{Égypte/Monde arabe}

$19 \mid 2019$

Les zabbâlîn, un objet surétudié ?

\title{
Interview avec la réalisatrice Julia Varga
}

Propos recueillis par Gaétan du Roy et Natalia Duque le 10 juillet 2017

An interview with filmmaker Julia Varga, by Gaétan du Roy et Natalia Duque

(10 ${ }^{\text {th }}$ of July 2017)

\section{Julia Varga}

\section{OpenEdition}

\section{Journals}

Édition électronique

URL : https://journals.openedition.org/ema/4537

DOI : $10.4000 /$ ema. 4537

ISSN : 2090-7273

Éditeur

CEDEJ - Centre d'études et de documentation économiques juridiques et sociales

Édition imprimée

Date de publication : 1 janvier 2019

Pagination : 51-60

ISBN : 9782900956052

ISSN : $1110-5097$

Référence électronique

Julia Varga, «Interview avec la réalisatrice Julia Varga », Égypte/Monde arabe [En ligne], 19 | 2019, mis en ligne le 01 janvier 2021, consulté le 07 juillet 2022. URL : http://journals.openedition.org/ema/4537 ; DOI : https://doi.org/10.4000/ema.4537 


\title{
Julia Varga
}

\section{INTERVIEW AVEC LA RÉALISATRICE}

\author{
JULIA VARGA
}

Propos recueillis par Gaétan du Roy et Natalia Duque le 10 juillet 2017

RÉSUMÉ

Julia Varga a filmé durant de longues années au Muqattam. Elle a suivi plusieurs personnages qui ont souhaité s'investir dans la vie de leur quartier après la révolution de 2011. Elle raconte dans cet entretien sa rencontre avec le Muqattam et ses habitants et réfléchit aux raisons qui expliquent pourquoi ce lieu attire tant de chercheurs, artistes et écrivains. Julia Varga dissèque avec beaucoup de lucidité l'attractivité du lieu et se positionne par-rapport aux représentations qui circulent sur les zabbâlîn. 


\section{Gaétan du Roy (GdR)}

Comment as-tu connu le quartier des zabbâlîn et comment es-tu arrivée là la première fois?

\section{Julia Varga (JV)}

Je suis arrivée là parce que l'école des Beaux-Arts m'avait envoyée à Beyrouth pour faire une résidence d'un mois. Je n'y connaissais rien et c'était une grande découverte. J'ai passé un mois là-bas. Et à partir de là, le Monde Arabe a commencé à m'intéresser.

J'ai ensuite rencontré Anna Roussillon ${ }^{1}$ par hasard alors que je revenais à peine de Beyrouth. Elle m'a dit qu'elle avait grandi en Égypte et je lui ai proposé qu'on y aille ensemble. C'était en 2003 mais j'ai fini par y aller seulement en 2007. J'étais dans une période où je touchais à tout, des peintures, des sons, et d'autres choses... J'ai beaucoup marché au Caire pour découvrir la ville. Anna m’a emmenée dans la Cité des morts mais ça ne retenait pas mon attention. Par contre depuis ce quartier, je regardais de l'autre côté de l'autoroute [vers Manshiyyat Nasir, ndlr] ces terrasses peintes individuellement qui m'ont beaucoup intriguée. À l'époque, je ne me rendais pas compte que des familles entières possédaient les immeubles.

J'ai voulu aller voir et nous sommes montées dans le quartier, d'abord dans la partie musulmane. On y est allé durant plusieurs jours puis on a été voir de I'autre côté près de la falaise du Muqattam. Et là, on a atterri chez les zabbâlîn dont je n'avais jamais entendu parler. Je ne savais même pas qu'il y avait des chrétiens en Égypte. Cela constitua une espèce de grand choc visuel pour moi. J'ai tout de suite été intéressée par le travail effréné qui s'y déroulait. J'étais impressionnée par l'ampleur du boulot : tout le monde s'acharnait à la tâche. Lorsque je suis arrivée, c'était en plein jour, au milieu d'une journée de travail : il y avait le bruit, le monde qui circule, les charges de matériel sur les camions, les ateliers... Là, je vois des gars torse-nu, avec des tatouages de la Vierge et de Jésus qui triaient minutieusement les bouchons de jus en plastique par couleur. Ça m’a rappelé les tatouages des prisonniers en Roumanie! C'était une fourmilière qui recycle des masses immenses de déchets du Caire. II y a de petits zabbâlîn sur des chariots brinquebalants en bois tiré par leurs ânes à côté des gros camions chargés de sacs tellement haut que le bout touche le deuxième étage des immeubles. Les bâtiments étaient éternellement en construction, avec sur les toits des élevages d'animaux, et des matières diverses stockées en attendant d'être recyclées. Le rapprochement de ces images m'a impressionnée. Puis j'ai vu le monastère là-haut. Et là, mon intérêt s'est cristallisé. C'est-à-dire que le rapport entre le bas et le haut avec cet espace new age, qui domine tout, spectaculaire et un peu de mauvais goût... Alors qu'en bas, on

1 Anna Roussillon est documentariste, elle a notamment réalisé le documentaire Je suis le peuple (2014) sur la révolution égyptienne de 2011. 
évolue comme enveloppé par les odeurs, les fumées : c'était spectaculaire pour moi. À l'époque je faisais des photos et je me suis dit : "si tu fais n'importe quoi ici, ça sera intéressant de toute manière ! ».

\section{GdR}

Ça stimule les sens?

\section{JV}

Tous les sens en effet. C'était donc très excitant. Ce qui m'intriguait vraiment c'était le rapport entre le pouvoir religieux, le déchet et la minorité religieuse dans un régime politique ou le président est président à vie (à l'époque Moubarak). Il y avait énormément d'ingrédients. Et j'avais l'impression que sur ce petit territoire, il y avait de nombreuses questions qui pouvaient être examinées. Des questions qui traversent l'Égypte plus globalement. J'avais l'impression que c'était un endroit exceptionnel pour observer l'ensemble.

\section{GdR}

Et donc, tu as commencé à filmer après la révolution?

\section{JV}

En 2010, nous avons filmé avec Anna. Mais je n'ai pas filmé mon personnage principal, Talaat, parce que je n'étais pas encore à l'aise pour sortir la caméra. Puis le grand frère de Noura [l'épouse de Talaat, ndlr] est soudain décédé d'un accident de voiture. Ce deuil ne permettait pas de filmer à ce moment-là. J'ai donc seulement commencé à filmer en 2010. Jusque-là j'avais pris des photos au Muqattam et dans d'autres quartiers de chiffonniers. J'ai commencé à lire ce qui avait été écrit sur le sujet. Entretemps j'ai vu le film Garbage Dreams.

\section{GdR}

Qu'as-tu pensé de Garbage Dreams et de la manière dont le film représentait ce quartier?

\section{JV}

Le film proposait de très jolies images du quartier, mais c'était une sorte de conte de fées. Il y avait beaucoup de clichés, on restait dans les stéréotypes. II y a des jeunes qui veulent s'en sortir mais ils en sont empêchés par la société et les discriminations et ils bravent tous les obstacles. Après, vient la confrontation avec l'Occident [les jeunes du quartier qui visitent une usine de recyclage au Pays de Galles se rendent compte qu'ils recyclent davantage au Caire avec des moyens rudimentaires, ndlr] où finalement, ils peuvent dire « Nous, on fait mieux qu'eux ». C'était une approche un peu sentimentaliste pour faire vendre, un peu cousue de fil blanc. C'était aussi un point de vue très occidental, un certain type de lecture de cet endroit qui marche bien pour les Occidentaux, très lisible. En résumé, je n’ai pas trop aimé, tout en reconnaissant ses qualités 
qui sont de l'ordre de l'artisanat. C'est un film bien fait dans son genre. Il fonctionne bien pour ce qu'il est destiné à faire. Ça a ému tout le monde...

\section{GdR}

Il y a un côté " pensée positive » aussi. "Regardez, à travers le monde, ces gens qui font des choses qui vont dans le sens de l'écologie, etc. »

JV

Tout à fait. Mais ça marche à différents niveaux. C'est joli, c'est bien fait et ça se défend. En le voyant je me suis dit que « tout » reste à faire - ce film « carte postale » ne nous amène pas très loin, ne tire même pas un des fils possibles (il y en a tellement dans le quartier).

\section{GdR}

Et tu t'es rendue progressivement compte que le quartier avait une certaine célébrité, qu'il attirait l'attention?

\section{JV}

Oui, très vite. Déjà, en vous rencontrant. D'ailleurs, en le découvrant je me suis dit que ce lieu devait être extrêmement connu. Or à l'époque, en 2007, on ne trouvait pas grande chose, curieusement. Ça m'a étonnée. Je n'avais pas encore connaissance des films. Je trouvais ce lieu tellement particulier, tellement haut en couleurs que je me disais que le monde devrait s'y intéresser. Après j'ai découvert progressivement qu'il y avait beaucoup de recherches, et quelques films tournés dans ce quartier. Certains qui s'y intéressaient semblaient vouloir protéger le sujet, ils voulaient montrer que c'était leur territoire mais cela ne $\mathrm{m}^{\prime}$ a pas intimidée pour y faire quelque chose.

Quand j'ai entendu que Garbage Dreams était sorti et avant de l'avoir vu, j'ai eu peur que ce film ait épuisé le sujet. Après la révolution, j'ai enfin trouvé le film qui était assez difficile à se procurer et j'ai fait une projection chez Talaat au Muqattam. On a invité du monde. Ce qui est très intéressant avec ce film, c'est qu'ils font tout pour que le quartier ne le voie pas. Je ne sais pas pourquoi, parce que quand je l'ai projeté, il n'y avait aucune animosité. Les gens l'ont regardé : ils étaient en train de crier " voilà untel, un autre... bien sûr, c'est lui! ». Et après, ils étaient plutôt flattés parce que c'était joli.

\section{GdR}

En 2010, tu travailles sur le quartier et tu commences à le filmer. Tu connais déjà les films qui y ont été faits. Est-ce que tu arrives à ne pas du tout en tenir compte? Ou est-ce tu es influencée par cela, ne serait-ce que par la volonté de te distancier de ces films? 
JV

J'ai compris que si je suivais juste mes envies, ça devrait nécessairement être différent. Je savais que cela allait être singulier, car je n'avais pas une approche thématique du sujet et que je ne voulais pas faire un film sur les zabbâlîn, ni un film sur le recyclage, ni quoi que ce soit de ce genre. En règle générale, les gens font un film «sur... ». Une fois que j'avais fait Check Check Poto ${ }^{2}, \mathrm{c}^{\prime}$ était plus facile pour moi, parce que j'avais déjà fait un film et que j'avais développé une façon de faire. Bien sûr les images typiques - c'est-à-dire les jeux faciles sur les contrastes entre de jolis enfants qui jouent sur des tas d'ordures ou les plans de femmes qui trient les déchets à mains nues - m'étaient restées dans I'œil, mais je ne les aurais de toute façon pas reproduites! Dans les images récentes que j'ai faites, j'essaye d'éviter ces manières stéréotypées de représenter le quartier. Moi j'ai filmé des réunions, des discussions... Au final cela n’a pas grand-chose à voir avec ce qui avait été fait.

\section{GdR}

Au fond, beaucoup de choses qui pourraient se passer...

\section{JV}

...ailleurs. Mais je trouvais ça très intéressant, justement, ce mélange entre les particularités locales et des aspects plus généraux que l'on retrouve ailleurs. Je ne veux pas les enfermer dans une sorte de thématique, du genre « eux, ce sont les zabbâlîn » qui insisterait lourdement sur leur identité de chiffonniers.

\section{GdR}

Une des choses à laquelle nous essayons de réfléchir dans ce numéro de revue, sont les raisons pour lesquelles ce quartier fascine et attire toutes sortes de gens : chercheurs, cinéastes, journalistes, etc. On est tous un peu attirés comme tu le disais au début. Ensuite cet attrait, on peut arriver à le maîtriser ou non. Mais, quels en sont les ingrédients selon toi? C'est la montagne? Les déchets? Le contraste entre le monastère et le reste du quartier?

\section{JV}

Il y a tout ça, mais il y a aussi le fait que tous le gens dont tu parles, qui se sont intéressés aux chiffonniers (pas tous, mais la plupart), sont des chrétiens ou en tout cas viennent de pays de tradition chrétienne. Et cela n'est pas du tout anodin. Quand on a fait, je ne sais pas combien d'années de catéchisme et qu'on est passé par l'Église, comme moi, on ne peut pas rester neutre en voyant des chrétiens.

2 Check Check Poto (2010) est un documentaire réalisé par Julia Varga sur le centre d'accueil des jeunes adolescents « Mosaïque » à Aubervilliers (France). 


\section{GdR}

Tu penses que cela joue un rôle aussi central?

\section{JV}

Oui, bien sûr. J'en suis persuadée. J'ai fouillé pendant des années entières des bibliothèques d'histoire de l'art au cours de mes années de formation et je me suis imprégnée de toutes ces représentations religieuses, ces images bibliques. Dès lors quand je vois au Muqattam des tatouages qui réinterprètent ces représentations, je ne peux pas rester insensible ! Cela crée un décalage qui m'intéresse. J'ai une familiarité avec le christianisme qui a fait partie de ma vie, or j'ignorais lors de cette première visite au Muqattam qu'il y avait des chrétiens en Égypte. D'une certaine manière le fait de retrouver des références familières à cet endroit où je ne $m^{\prime} y$ attendais pas du tout $m^{\prime} a$ émue. Et donc j'ai donc eu envie de comprendre et de voir quelles sont leurs façons de vivre leur christianisme... D'une certaine manière, je les reconnais comme étant proches. Je n'en parle pas, mais émotionnellement cela joue un rôle. Mais comme je ne revendique pas d'être de religion chrétienne, c'est quelque chose dont je ne discute pas, je ne le mentionne pas. Cela ressort juste parce que je parle avec vous. Je pense que ça a dû probablement être le cas de la plupart des Occidentaux qui se sont intéressés aux chiffonniers : même s'ils ne le reconnaissent pas (ils sont peut-être athées) cet élément crée une sorte de lien. D'ailleurs les gens nous voient souvent comme de potentiels alliés, parce qu'ils nous perçoivent comme des chrétiens.

\section{Natalia Duque (ND)}

Tu t'intéresses aux coptes parce qu'ils ont une façon spécifique d'être chrétien ? Ou parce que tu te retrouves en eux?

JV

Parce qu'on soupçonne une hostilité autour d'eux et on se solidarise. Parce qu'ils sont des nôtres, un peu, quelque part. Et on veut communier avec eux, être en contact, les aider en quelque sorte.

\section{GdR}

Et en plus, ils sont mal vus, parce qu'ils sont des zabbâlîn...

\section{JV}

Exactement. Et il y a aussi notre éducation. C'est-à-dire que si on a une éducation un peu chrétienne, on nous apprend à aider ceux qui sont en difficulté, tu vois. Je crois que ces traditions dépassent la pratique religieuse dans nos sociétés. On nous apprend des valeurs comme l'amour de l'autre, l'entraide...

Il faut dire qu'en tant qu'enfant j'ai moi-même grandi comme Hongroise en Roumanie. On avait notre langue et notre appartenance a été importante lors notre socialisation - je vis en France aujourd'hui, donc en quelque sorte libérée 
de ce "poids », mais plus que le christianisme des coptes d'Égypte, ce qui les rapproche de mon expérience c'est d'être une minorité (même s'ils refusent souvent qu'on leur attribue ce qualificatif) au sein d'un État musulman habité majoritairement par des musulmans. Je pense que c'est pesant de faire partie d'une minorité qui tente de préserver sa " différence », on est comme obligé de prendre en charge cette préservation.

En Roumanie, on était dans une dictature et donc, il y avait pour moi une sorte de réplique de cette situation sur un autre territoire. Cela m'a intriguée parce que je pouvais observer la même chose mais ailleurs que chez moi ! Chez toi, c'est compliqué, tu appartiens à un camp. Ici tu peux avoir une plus grande distance ... Si tu arrives de l'extérieur, tu as toujours plus de recul. Donc, cela m'a permis d'observer des phénomènes que je connaissais depuis mon enfance, mais ailleurs.

Après, il y a aussi l'aspect visuel. J'ai fait les Beaux-Arts, donc, j'ai passé des années à faire des dessins, des peintures et après des photos, et visuellement ce quartier est assez spectaculaire... Comme tout le reste du Caire, d'ailleurs! Cela m'a intriguée aussi parce que déjà, on était un peu préoccupé par la pollution à l'époque et par les poubelles et tout ce qu'on produit et que c'était une sorte de mise en scène de la misère du monde telle qu'elle est orchestrée économiquement, en terme de production de plastiques, de déchets... Quelque part, c'était un endroit qui offrait une sorte de critique de la réalité à plusieurs points de vue : la dictature, le traitement réservé aux minorités, et toute cette histoire autour des déchets, de la pauvreté...

\section{GdR}

J'ai toujours l'impression que le Muqattam c'est un peu un "village en ville". Il y a un côté villageois, parce que tu circules dans les petites ruelles, les gens sont assis devant leurs maisons...

\section{JV}

Et cela souligne les contrastes. Tu es dans une métropole énorme, et, en même temps, dans un village : tu es dans un grand pays musulman, mais tu es chez les chrétiens. Tout le temps, il y a des contrastes. Il y a des contrastes à l'intérieur du quartier, entre le monastère et les rues. Contrairement à ce qu'on pourrait attendre, le fait que ce soit un endroit sur lequel on a fait beaucoup de recherches et de films ne m'a pas empêché de m'y intéresser. Cela m'a encore plus intéressée parce que j'ai trouvé stimulant le rapport entre les différentes approches et les différents films. Et donc, j'avais envie de proposer une autre façon de montrer les choses, justement parce qu'on l'avait déjà vu autrement.

D'ailleurs la célébrité du quartier ne m'a pas du tout aidée au début, au contraire. La chaîne Arte a dit tout de suite " cela ne nous intéresse pas, cela a déjà été fait ». Dans le milieu artistique où j'évolue, ils en ont marre des films de poubelles et donc ça ne les intéressait pas. De leur côté les Égyptiens détestent ça parce que ça donne une mauvaise image du pays. Et d'ailleurs 
Qutaiba, mon monteur m'a conseillé de ne plus dire que je faisais un film sur les zabbâlîn mais plutôt que je faisais un film sur un avocat et un type de l'ancien régime. C'était magique! Tout le monde était intéressé.

\section{GdR}

Ton film est parti d'une rencontre, avec la famille de Talaat, un avocat $d u$ quartier.

\section{JV}

En effet la rencontre avec Talaat a été cruciale parce que c'est quelqu'un d'une grande tendresse. En plus il est intelligent, motivé et, en même temps, il a une posture inouïe dans le quartier. II est très généreux de tout ce qu'il sait, de tout ce qu'il pense, de ses sentiments : il raconte, il t'accueille. Je l'ai trouvé très singulier. C'était la première personne, la seule d'ailleurs qui m’ait vraiment ouvert son cœur, qui avait envie de faire un film aussi. Il espérait que ce film lui rendrait justice. Il avait envie que justice soit rendue, c'est ça qui lui a donné envie de faire ce film. Très longtemps, il en a été le véritable co-auteur. Il faisait des mises en scène pour le tournage. Il conviait les gens, il les questionnait avec la volonté de montrer la réalité. Mon envie de filmer a rencontré cette envie qui venait de lui.

\section{GdR}

Est-ce que la Révolution a joué un rôle dans l'envie de Talaat de dire des choses, de rendre justice?

\section{JV}

Avant même la Révolution, il m’avait encouragée à filmer. Il me disait « tu vas leur dire que tu fais des images pour toi, ce n'est pas grave, rien ne se passera, vas-y, filme ! ». C'était en 2010. Simplement, après la Révolution, il s'est mis en mouvement beaucoup plus intensément, et il y avait de la matière parce que les revendications locales éclataient au grand jour, et donc, effectivement, cela l'a motivé mais l'envie était déjà là.

II voulait agir. Il a sans doute confondu le film avec l'action. Au point que quand il s'est détaché de tout, il est tombé dans une espèce de dépression, à un moment donné, il est rentré, j'étais chez lui, assise, et il m’a dit " mais qu'est-ce qu'on va faire maintenant? Le film, tout ça... est raté. Tout est raté ». Je lui ai dit «Tu sais, les films sont résistants, ils résistent à tout, et des fois, ils sont intéressants justement parce que tout est raté ». Les films peuvent surmonter les ratages, même les pires, parce qu'ils les racontent justement. Et donc, du coup, le film, on ne le perd pas, par contre, derrière, on peut souffrir.

\section{GdR}

Réussir la Révolution pour réussir le film... 
JV

Exactement. II fallait que lui réussisse. Pas seulement la Révolution, mais lui, en tant que personnage principal, il se devait de réussir. Il devait réussir l'association $^{3}$. Et pour moi c'était une grande aventure. J'ai toujours dit que d'avoir tourné ce film, en soi, même s'il n'y a pas de film à la fin, cela valait le coup. Et je me suis déjà dit ça il y a quelques années : une fois que j'étais un peu plus mûre. Je me suis dit « ça ne sert à rien de faire quoique ce soit, surtout pas des films, si, déjà rien que le tournage ne vaut pas le coup en soi ».

\section{GdR}

On crée des liens qui vont au-delà du film.

\section{JV}

C'est ça! Mais aussi cela te fait évoluer parce que tu vois des choses qui te font réfléchir. Et tu participes en fait à ce réel. C'est-à-dire, qu'en tant que cinéaste, tu actives aussi ce réel, tu y joues un rôle. Et donc, même si tu es juste là, en train de filmer, et qu'il n'y a pas de bobine dedans, mais que tu ne le sais pas, ni toi, ni les autres, ce n'est pas grave. Il se passe autre chose parce que tu es là. Il est arrivé que les membres de I'association [à laquelle participait Talaat, ndlr], me disent après coup : «En fait, on a créé l'association en partie parce que tu étais là ». À un moment c'était presque ça ! "Parce que tu nous as emmenés voir la "shirka X »["shirka » signifie compagnie ou société en arabe, ici une des compagnies étrangères chargée de ramasser les déchets au Caire, ndlr], et que son représentant nous a demandés qui on était? Et on s'est rendu compte qu'on n'était rien et donc on s'est dit qu'il fallait créer une association pour exister».

\section{GdR}

Tout ce que tu dis à propos du rôle du cinéaste, de l'importance que cela a pour toi, des évolutions que cela provoque... Cela fait penser un peu à l'anthropologie. Te sens-tu un peu comme une anthropologue avec une caméra?

\section{JV}

Très proche ! Je suis une anthropologue détournée, pervertie, une mauvaise anthropologue, très mauvaise, puisque je ne suis aucune règle préétablie parce que je ne suis pas très méthodique, parce que je picore à gauche et à droite au lieu de prendre des notes, de réfléchir correctement, donc je suis une très mauvaise anthropologue ! Par contre j'ai une espèce d'appétit forcené

3 Talaat voulait mettre en place une association dans le quartier pour servir les habitants. Cette histoire est racontée dans Gaétan du Roy, "La campagne d'al- Misriyyîn al-Ahrâr chez les chiffonniers de Manshiyit Nâser », in Egypte/Monde arabe, n 10, $3^{\text {e }}$ série, 2013 : STEUER C. (dir.), Les élections de la révolution. 
pour fouiller, pour ne jamais m'arrêter sur ce qui serait bien pour le scénario ou ce qui sonnerait bien... J'ai toujours beaucoup d'intérêt pour comprendre ce qu'il y a derrière les apparences. C'est le contraire de Garbage Dreams, quelque part.

\section{GdR}

Garbage Dreams est très scénarisé, cherchais-tu aussi à ne pas trop écrire ton film? À observer les choses comme elles viennent?

\section{JV}

Exactement. Et je ne peux pas faire ça. Ce qui m'intéresse justement, c'est de ne pas savoir et puis d'apprendre au fur et à mesure. En ça, je suis assez puriste, c'est-à-dire que je présume que ma compréhension des situations va se développer, changer, parce que je vais passer tout ce temps là-bas. Et c'est pour cela que j'y vais. Je me dis que là, je vois ça, mais «ça » $c^{\prime}$ est juste la surface. Et de là, avec mes stéréotypes, avec ma connaissance actuelle, je vais me déplacer et je vais comprendre des choses. 